\title{
Attainable Landing Area Computation of a Lunar Lander with Uncertainty by Reachability Analysis
}

Yunus Emre Arslantas and Stephan Theil

\begin{abstract}
Soft landing is one of the most critical phases for space missions which require landing a spacecraft on the surface of a body like asteroids or planets, as well as concepts like reusable launch vehicles. In order to ensure safety and success for the terminal landing phase, in addition to hazard maps obtained by on-board sensors, there is also a need for a map which characterizes the attainable landing area that the lander can achieve by obeying constraints within the presence of uncertainties. This paper proposes a method to obtain the attainable landing area of a lunar lander with uncertainties by reachability analysis. The method obtains the set of achievable states for a dynamical system starting from an initial condition with given admissible control inputs of the system.

Nonconvex reachable sets (RS) are computed using optimal control. The candidate landing area on the Moon surface is represented by equidistant grid points and for each point an optimal control problem (OCP) is defined. The corresponding OCP is transcribed into a finite dimensional Nonlinear Programming Problem (NLP) by using Pseudospectral Methods (PSM). The solution of the NLP leads to the RS of the dynamical system. A Riccati equation-based controller is designed to track the reference trajectories. Monte Carlo simulations are carried out to obtain the safely attainable landing area of the lunar lander as probability maps.
\end{abstract}

Yunus Emre Arslantas

Department of Mathematics and Computer Science - University of Bremen,

28359 Bremen/Germany

e-mail: Yunusemr@uni-bremen.de

Stephan Theil

DLR Institute of Space Systems, Robert-Hooke Str. 728359 Bremen/Germany

e-mail: Stephan.Theil@dlr.de 


\section{Introduction}

In recent years, advances of the proven technological heritage and new sophisticated tools led to more challenging space missions. Along with the government initiatives, private companies also increase their efforts for space exploration and exploitation. As a result of these contributions, concepts like reusable launch vehicles, asteroid mining and establishing bases on extraterrestrial space bodies are getting closer to reality. State of the art guidance and control algorithms are required for the success of these missions. In particular, the determination of hazards, the implementation of landing maneuvers and maximizing the likelihood of a safe landing is of paramount importance.

From the safety point of view it is important to ensure that the target landing area is suitable for landing due to surface properties and that is also within the reach of the lander. At this point two sets of information are important:

- Hazard Maps: obtained by optical sensors and contain information about the craters, the roughness of the landing surface and the slope of the terrain.

- Attainable Map: related to the physical limits of the lander like available onboard propellant, state constraints (final attitude and velocity for safe landing) and control constraints (avoiding saturation of controllers).

This paper proposes an algorithm to obtain the attainable map of a lunar lander for a generic lunar mission scenario using reachability analysis. Reachability deals with the problem of whether from a given point, a system will eventually be able to reach another given point as illustrated in Fig. 1. Reachability analysis could either be used for verification or determination of the safety of a system (like computation of the safe landing envelope of an aircraft [1], the landing corridor of a reusable launch vehicle or reentry problems $[2,3,4]$. The reachability problem is handled by set-valued methods [5], level-set methods [6] or OCP based methods [7].

Fig. 1: Attainable Landing Area for a Lunar Lander

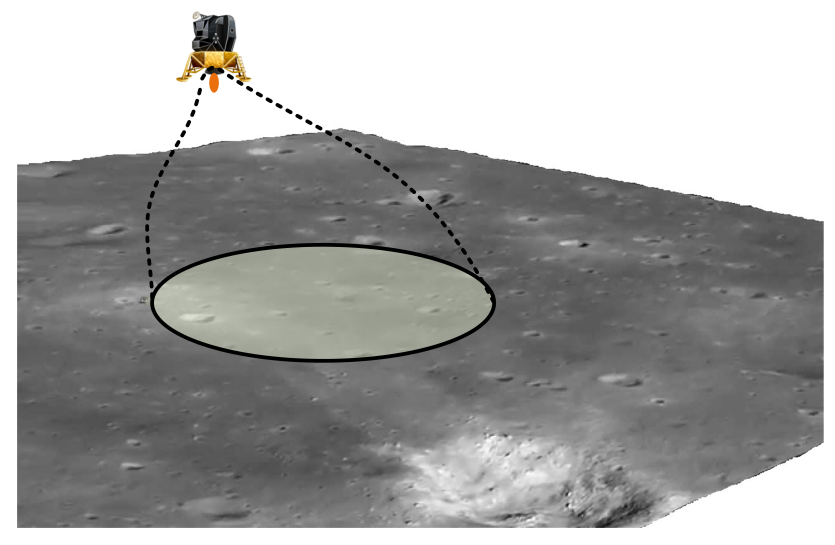


In order to obtain the reachable set (RS) of the lunar landing problem, the candidate landing area is characterized by discrete equidistant landing points. For each point a distance function, which acts as the objective function of an associated optimal control problem (OCP), is defined. These infinite dimensional OCPs are transcribed into a Nonlinear Programming (NLP) problem using Pseudo-Spectral methods (PSM).

PSM uses a linear combination of Legendre polynomials to obtain discretization points or nodes. This methodology could be classified into different groups according to the used discretization points, namely Legendre-Gauss, Legendre-GaussRadau or Legendre-Gauss-Lobatto [8]. In these approaches, the continuous functions are collocated using the corresponding nodes, leading to a sparse structure of the Jacobian. Finally the NLP is solved with commercial off-the-shelf solvers to obtain the state information at the landing points. The union of the points approximate the reachable landing area with an error, due to the discretization of the domain.

In order to verify the effect of uncertainties, which could be encountered during the mission phase, Monte Carlo simulations are performed. The main source of uncertainties are the initial state or system parameters. Later on, the probabilistic attainable maps are obtained by counting the number of successful landings on the candidate points. The rest of the paper is organized as follows:

Section 2 defines basic terms associated with reachability analysis. Section 3 describes the dynamical model of the lunar lander including the mission scenario and the associated constraints. Section 4 presents an optimization based methodology for the computation of reachable sets, the discretization of the infinite dimensional OCP and the design of the controller. Monte Carlo simulations with uncertainties and probabilistic attainable maps are discussed in Section 5. Finally Section 6 concludes the paper with a short summary.

\section{Reachability}

Consider a dynamical system with:

$$
\begin{aligned}
\dot{x}(t) & =f(x(t), u(t), t), \quad t \in \mathbb{I}=\left[t_{0}, t_{f}\right], \\
x\left(t_{0}\right) & =x_{0} \in X,
\end{aligned}
$$

where $X \subset \mathbb{R}^{n}$ denotes the state space and $U \subset \mathbb{R}^{m}$ the input space. $x_{0}=x\left(t_{0}\right)$ is the state at initial time $t_{0}$ while $x_{f}=x\left(t_{f}\right)$ denotes the final state. The control input is contained in the space of admissible control signals, that is $u(t) \in \mathscr{U}=$ $\mathrm{L}^{\infty}\left(\left[t_{0}, t_{f}\right], U\right)$. For each point in time, the solution or state trajectory $x(\cdot): \mathbb{R}_{+} \rightarrow X$ of (1) is an element of the state space $x(\cdot) \in X$.

Definition 1. Given $x_{0}$ and the set of all admissible $\mathscr{U}$, the reachable set of (1) at time $t_{f}$ is defined as 
$\mathscr{R}_{t f}\left(x_{0}\right)=\left\{x_{f} \in X \mid \exists u \in \mathscr{U} \wedge \exists x(\cdot) \in X\right.$ s.t. (1) holds $\left.\wedge x_{f}=x\left(t_{f}\right)\right\}$.

In other words, a reachable set includes the set of final states of a given dynamical system for a final time and given constraints.

In order to determine an approximated RS, the two dimensional subset of the state space $G_{s}$ is discretized by equidistant grid points $g_{s}$ as illustrated in Fig. 2 .

Fig. 2: Discretization of the Landing Area with Uniform Grid Points.

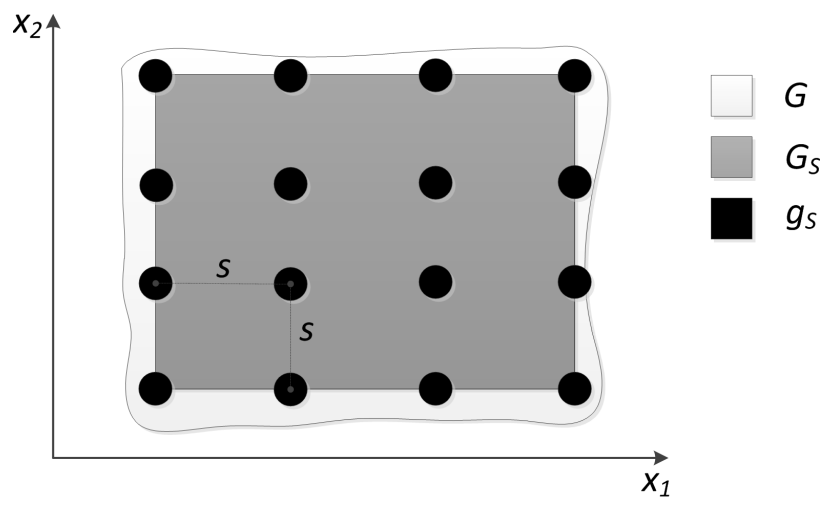

The OCP defined by (3) is solved to find control inputs that steer the system from the initial state to a sufficiently small neighborhood of a corresponding grid point $g_{s}$. If an optimal solution is found, the point is said to be reachable. Otherwise, if the solution cannot be found, the candidate point is excluded from the set. Instead, final states of the associated problem are included in the set. In other words, the solution which minimizes the distance between the candidate landing point on crossrangedownrange plane and final states obtained from the solution of NLP are taken into account. The set which encloses all these final points constitutes the approximated RS. The OCP is formulated as

$$
\begin{aligned}
\min _{u \in \mathscr{U}} \quad \frac{1}{2}\left\|x\left(t_{f}\right)-g_{s}\right\|_{2}^{2} \\
\dot{x}=f(x(t), u(t)), \text { a.e. in }\left[t_{0}, t_{f}\right] \\
x_{0} \in X_{0} \subseteq \mathbb{R}^{\mathrm{n}}
\end{aligned}
$$

with inequality constraints

$$
\begin{aligned}
& x_{L} \leq x(t) \leq x_{U} \\
& u_{L} \leq u(t) \leq u_{U}
\end{aligned}
$$

where $\dot{x}=f(x(t), u(t))$ is the equality constraint to be satisfied. For a given $x_{0}$, let $u^{\star}\left(\cdot ; g_{s}\right)$ be the solution of (3), and $x^{\star}\left(\cdot ; g_{s}\right)$ is the corresponding solution of the underlying system dynamics. Hence the approximation of the reachable set is defined as 


$$
\mathscr{R}_{t_{f_{s}}}\left(x_{0}\right)=\bigcup_{g_{s} \in G_{s}}\left\{x^{\star}\left(t_{f} ; g_{s}\right)\right\} .
$$

where $t_{f} \in \mathbb{I}$. State information at $t_{f}$ are used to obtain an approximation of the RS.

\section{Dynamical Model and Mission Scenario}

The equations of motion of the lunar lander are taken from [9]. However, instead of a non-throttable main engine, the thrust level is modulated. Moreover, orthogonal thrusters are discarded in the model. Only one main engine provides thrust during the final phase of the landing, leading to following equations of motion

$$
\left(\begin{array}{l}
\dot{d} \\
\ddot{h} \\
\ddot{c}
\end{array}\right)=\left(\begin{array}{c}
\frac{r}{m \tilde{h} \mathrm{c}(\tilde{c})}\left(-T_{1} \mathrm{c}(\tilde{d})+T_{2} \mathrm{~s}(\tilde{d})\right)+2 \dot{d}\left(\frac{\dot{c}}{r} \mathrm{t}(\tilde{c})-\frac{\dot{h}}{\tilde{h}}\right) \\
\frac{1}{m}\left[\left(-T_{1} \mathrm{~s}(\tilde{d})-T_{2} \mathrm{c}(\tilde{d})\right) \mathrm{c}(\tilde{c})-T_{3} \mathrm{~s}(\tilde{c})\right]+\left[(\dot{d} \mathrm{c}(\tilde{c}))^{2}+\dot{c}^{2}\right] \frac{(\tilde{h})}{r^{2}}-\frac{M G}{(\tilde{h})^{2}} \\
\frac{r}{m(\tilde{h})}\left[\left(T_{1} \mathrm{~s}(\tilde{d})+T_{2} \mathrm{c}(\tilde{d})\right) \mathrm{s}(\tilde{c})-T_{3} \mathrm{c}(\tilde{c})\right]-\frac{\dot{d}^{2}}{r} \mathrm{~s}(\tilde{c}) \mathrm{c}(\tilde{c})-\frac{2 \dot{c} \dot{h}}{\tilde{h}}
\end{array}\right)
$$

where $d$ is the downrange, $c$ is the crossrange, $h$ is the altitude and $m$ is the mass of the lander. In addition the following abbreviations are used in (6)

$$
\begin{aligned}
& \tilde{h}=h+r, \quad \mathrm{~s}(\tilde{d})=\sin \frac{d}{r}, \quad \mathrm{c}(\tilde{d})=\cos \frac{d}{r} \\
& \mathrm{~s}(\tilde{c})=\sin \frac{c}{r}, \quad \mathrm{c}(\tilde{c})=\cos \frac{c}{r}, \quad \mathrm{t}(\tilde{c})=\tan \frac{c}{r} \text {. }
\end{aligned}
$$

The transformed thrust vector is given by

$$
\left(\begin{array}{l}
T_{1} \\
T_{2} \\
T_{3}
\end{array}\right)=\left(\begin{array}{c}
\cos \left(\beta-\frac{d}{r}\right) T \cos _{\chi} \\
\sin \left(\beta-\frac{d}{r}\right) T \cos \chi \\
-T \sin _{\chi}
\end{array}\right)
$$

where $T$ is the modulated thrust value. The thrust vector is defined in non-dimensional form. The first derivatives of the pitch angle $\beta$ and yaw angle $\chi$ are also added to the system of differential equations, where $\omega_{\beta}$ and $\omega_{\chi}$ are the commanded angular rate

$$
\dot{\beta}=\omega_{\beta}, \quad \dot{\chi}=\omega_{\chi} .
$$

The thrust dependent fuel consumption, depending on $\sigma_{S F C}$, is defined as

$$
\dot{m}=-|T| \cdot \sigma_{S F C}
$$

All states of the dynamical system defined by $(6,8,9)$ are represented in the following state vector

$$
x=(\dot{d}, \dot{h}, \dot{c}, d, h, c, \beta, \chi, m)^{T}
$$


while the control vector is represented by

$$
u=\left(T, \omega_{\beta}, \omega_{\chi}\right)^{T} .
$$

\subsection{Mission Scenario}

The reachable sets for the lunar lander are obtained for the terminal landing phase of the mission. The spacecraft starts a landing maneuver from an altitude of $96 \mathrm{~km}$ and moves on an elliptical descent orbit with an altitude of $15 \mathrm{~km}$. Powered descent starts at this altitude. The main thruster reduces the velocity of the spacecraft until an altitude of $2 \mathrm{~km}$ is achieved. At the approach phase, an attitude adjustment maneuver is performed between $2 \mathrm{~km}$ and $300 \mathrm{~m}$. The final phase of the landing maneuver is supposed to start at an altitude of $300 \mathrm{~m}$ as shown in Fig. 3.

Fig. 3: Mission Scenario for the Powered Descent and Landing
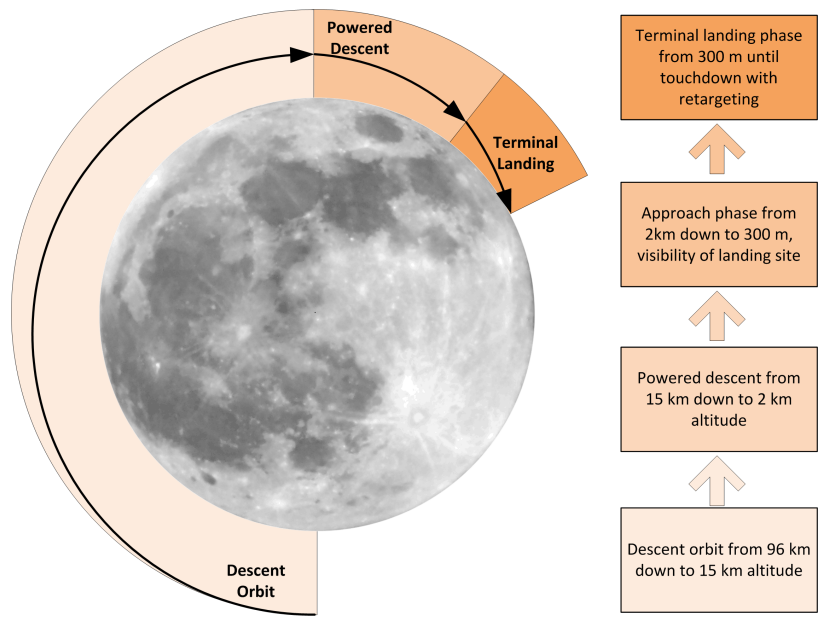

The initial conditions are defined by the state of the dynamical system at an altitude of $h_{0}=300 \mathrm{~m}$ using the optimal trajectory in [9]. The mission requires the following initial and terminal conditions

$$
\begin{aligned}
& x_{0}=\left(\dot{d}_{0}, \dot{h}_{0}, \dot{c}_{0}, \text { free, } h_{0}, \text { free }, \beta_{0}, \chi_{0}, m_{0}\right)^{\mathrm{T}} \\
& x_{f}=\left(0,0,0, \text { free, } 0, \text { free },-\frac{\pi}{2}, \text { free, } m_{f}\right)^{\mathrm{T}} .
\end{aligned}
$$

Additional constraints results from final landing condition. A landing is considered successful if the condition $\left|\Delta x\left(t_{f}\right)\right| \leq \Delta x_{\max }$ holds with

$$
\Delta x_{\max }=\left(1 \mathrm{~m} / \mathrm{s}, 1 \mathrm{~m} / \mathrm{s}, 1 \mathrm{~m} / \mathrm{s}, d_{f}, 1 \mathrm{~m}, c_{f}, 10^{\circ}, 180^{\circ}, \text { free }\right) .
$$


The initial conditions, final conditions and constraints are summarized in Table 1, where $x_{u}$ and $x_{l}$ denote upper and lower bounds on states respectively for the whole time interval. Similarly $u_{0}$ and $u_{f}$ represents initial and final control inputs, $u_{u}$ and $u_{l}$ are associated upper and lower bounds on the control inputs which are summarized in the Table 2.

Table 1 State Constraints used for the solution of OCP in (3)

\begin{tabular}{lllll}
\hline & $x_{0}$ & $x_{f}$ & $x_{l}$ & $x_{u}$ \\
\hline$\dot{d}(\mathrm{~m} / \mathrm{s})$ & 5 & 0 & free & free \\
$\dot{h}(\mathrm{~m} / \mathrm{s})$ & -19 & 0 & free & free \\
$\dot{c}(\mathrm{~m} / \mathrm{s})$ & 0 & 0 & free & free \\
$d(\mathrm{~m})$ & 0 & free & free & free \\
$h(\mathrm{~m})$ & 300 & 0 & 0 & free \\
$c(\mathrm{~m})$ & 0 & free & free & free \\
$\beta\left(^{\circ}\right)$ & -86 & -90 & -90 & 90 \\
$\chi\left(^{\circ}\right)$ & 0 & free & 0 & 180 \\
$m$ & 0.5397 & $\leq 0.5$ & 0.5 & 0.5397 \\
\hline
\end{tabular}

In this setup, the mass of the lander is the sum of the propellant mass and the mass of the structure. The allowed minimum mass of the vehicle is defined as hard constraint during trajectory optimization.

Table 2 Constraints on the Control Inputs for the solution of OCP in (3)

\begin{tabular}{lllll}
\hline & $u_{0}$ & $u_{f}$ & $u_{l}$ & $u_{u}$ \\
\hline$T$ & 0 & free & 0 & 1.222 \\
$\omega_{\beta}(\circ / \mathrm{s})$ & 0 & free & -2 & 2 \\
$\omega_{\chi}(\circ / \mathrm{s})$ & 0 & free & -2 & 2 \\
\hline
\end{tabular}

\section{Optimal Control Problem}

In this study the infinite dimensional OCP in (3) is converted into a corresponding finite dimensional NLP which is solved via a direct method. The basic principle is to collocate the differential equations, the cost functional and related constraints at a finite number of collocation points. After the continuous OCP is transcribed into a finite dimensional NLP, the resulting set of nonlinear algebraic constraints are solved numerically.

The collocation points are obtained using linear combination of the roots of Legendre Polynomials or their derivatives. The Gauss Pseudospectral Method (GPM), Lobatto Pseudospectral Method (LPM) and Radau Pseudospectral Method (RPM) 
with its flipped version (f-RPM) are the well known Pseudospectral methods for discretization of continuous problem with respect to the time domain [10, 11, 12]. Pseudospectral methods are advantageous due to:

- their straightforward implementation leading to a sparse NLP structure after discretization of the OCP;

- the avoidance of the Runge phenomenon [13];

- exponential convergence behavior for smooth problems [8];

In this study, a flipped variation (f-RPM) of the RPM is used to transcribe the OCP in (3). The roots of the linear combination of Legendre polynomials are obtained by RPM and flipped in the domain [-1,1]. SPARTAN (SHEFEX-3 Pseudospectral Algorithm for Reentry Trajectory ANalysis), an in-house developed trajectory generation tool, is used for the discretization of the problem $[14,15]$.

SPARTAN obtains the NLP by hybridization of the jacobian $\mathscr{J}$ by evaluating the different contributors as illustrated in Fig. 4 . The methodology is described in [16], and tested for the computation of the reachable sets in [17, 18].

Fig. 4: Hybridization and Representation of the Different Contributors of the jacobian $\mathscr{J}$ for the NLP.
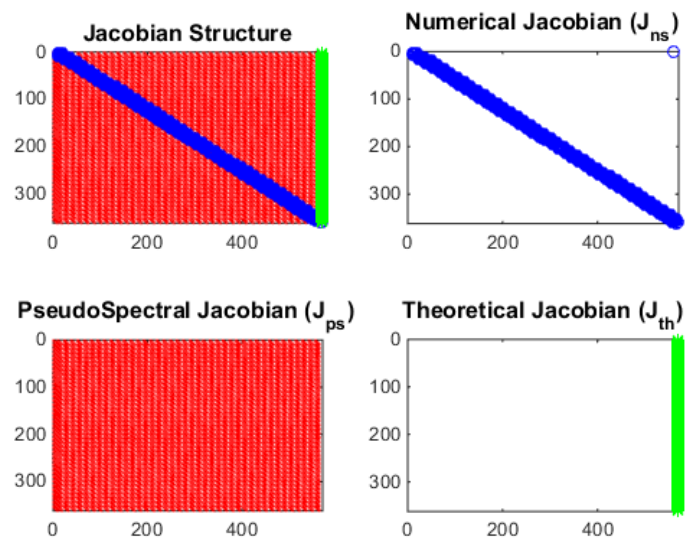

After solving the NLP of the associated OCP in (3), state information and control inputs for each grid point $g_{s} \in G_{s}$ are obtained. In addition to the exit flag of the solver, the result is also validated by propagating the control inputs by Runge-Kutta integration schemes and comparing it with the state information obtained within the same solution of the OCP. After the computations, the RS of the lunar lander is obtained including trajectories and control inputs for each of the related landing points with a final time $t_{f}=40 \mathrm{~s}$. 


\subsection{Feedback Controller}

The trajectory control of the lunar lander consist of a combination of feedforward and feedback controls as illustrated in Fig. 5.

For the purpose of the design of the feedback controller the dynamics of the lunar lander are represented by the nonlinear state space model as shown in (1). Assuming that the aforementioned state space representation is analytic and that a real landing trajectory is close to the optimal trajectory $\mathbf{x}(t)=\hat{\mathbf{x}}(t)+\delta \mathbf{x}(t)$, the linearization of (1) along $\hat{\mathbf{x}}(t)$ and $\hat{\mathbf{u}}(t)$ yields

$$
\dot{\delta} \mathbf{x}(t)=\mathbf{A}(t) \delta \mathbf{x}(t)+\mathbf{B}(t) \delta \mathbf{u}(t)
$$

with $\mathbf{A}(t)=\left.\frac{\partial \mathbf{f}}{\partial \mathbf{x}}\right|_{\hat{\mathbf{x}}, \hat{\mathbf{u}}} \in \mathbb{R}^{n \times n}$ and $\mathbf{B}(t)=\left.\frac{\partial \mathbf{f}}{\partial \mathbf{u}}\right|_{\hat{\mathbf{x}}, \hat{\mathbf{u}}} \in \mathbb{R}^{n \times m}, t \in\left[t_{0}, t_{f}\right]$ and corresponding initial conditions. Considering a time variant control law for the linear time variant dynamical system leads to

$$
\begin{aligned}
\delta \mathbf{u}(t) & =-\mathbf{K}(t) \delta \mathbf{x}(t) \\
\mathbf{u}(t) & =\hat{\mathbf{u}}(t)-\mathbf{K}(t) \delta \mathbf{x}(t), \quad t \in\left[t_{0}, t_{f}\right]
\end{aligned}
$$

Fig. 5: Block diagram of the closed loop system

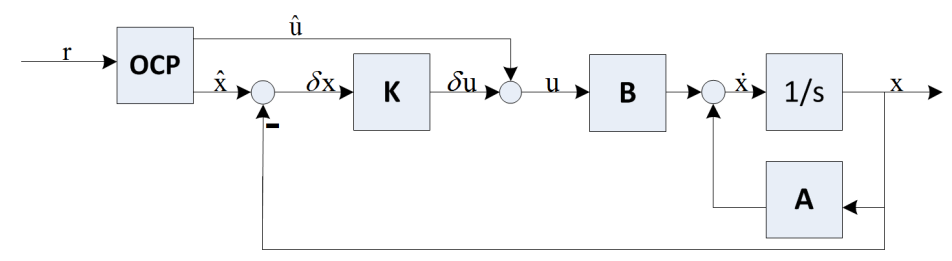

The changes in the dynamics of the lunar lander during a landing maneuver along a reference trajectory are supposed to be slow compared to the sampling rate of the discretized version of the OCP in (3). For this reason the gain matrix of the feedback controller $\mathbf{K}(t)$ is calculated only at each point $t_{i}, i \in \mathrm{I}$ with $\mathrm{I}=1, \ldots, l$ of the control discretization by minimization of the cost function

$$
\mathscr{J}(\delta \mathbf{x}, \delta \mathbf{u})=\int_{0}^{\infty} \delta \mathbf{x}(\tau)^{\mathbf{T}} \mathbf{Q}\left(t_{i}\right) \delta \mathbf{x}(\tau)+\delta \mathbf{u}(\tau)^{\mathbf{T}} \mathbf{R}\left(t_{i}\right) \delta \mathbf{u}(\tau) \mathrm{d} \tau
$$

for all $i \in \mathrm{I}$. Assuming the stabilizability of the underlying system, and positive semi-definite $\mathbf{Q}\left(t_{i}\right)$ and $\mathbf{R}\left(t_{i}\right)$ for all $i \in \mathrm{I}$, the feedback matrix that solves the OCP is given as a function

$$
\mathbf{K}\left(t_{i}\right)=\mathbf{R}\left(t_{i}\right)^{-1} \mathbf{B}\left(t_{i}\right)^{\mathrm{T}} \mathbf{S}\left(t_{i}\right), \quad i \in \mathrm{I}
$$


of the unique stabilizing solution $\mathbf{S}\left(t_{i}\right)$ of the algebraic Riccati equation

$$
\mathbf{A}\left(t_{i}\right)^{\mathrm{T}} \mathbf{S}\left(t_{i}\right)+\mathbf{S}\left(t_{i}\right) \mathbf{A}\left(t_{i}\right)-\mathbf{S}\left(t_{i}\right) \mathbf{B}\left(t_{i}\right) \mathbf{R}\left(t_{i}\right)^{-1} \mathbf{B}\left(t_{i}\right)^{\mathrm{T}} \mathbf{S}\left(t_{i}\right)+\mathbf{Q}\left(t_{i}\right)=0, \quad i \in \mathrm{I},
$$

such that the eigenvalues of $\mathbf{A}\left(t_{i}\right) \mathbf{B}\left(t_{i}\right) \mathbf{K}\left(t_{i}\right), i \in \mathrm{I}$ are negative. A linear interpolation has been applied to the $\mathbf{K}\left(t_{i}\right), i \in \mathrm{I}$ to obtain a time-continuous control law.

The main goal for the feedback control is to achieve the final landing point by following the reference trajectory while attenuating external disturbance or uncertainties for the initial state. The weighting matrices $\mathbf{Q}$ and $\mathbf{R}$ are kept constant during the simulations for each reference trajectory.

Fig. 6: Simulation of the Closed Loop System for the Tracking Reference Trajectories.
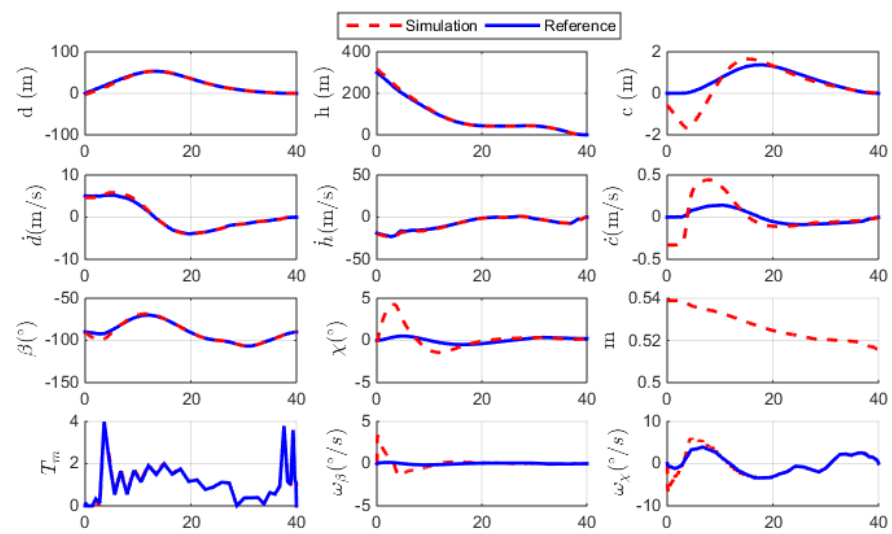

Fig. 6 shows the simulation of the closed loop system with initial disturbance. Similarly, Fig. 7 shows the discrepancy between the reference trajectory and the simulated states of the closed loop system. It can be seen from the plots that the controller is able to track the given reference trajectory by attenuating the initial state error.

Monte Carlo simulations are carried out to obtain the successful attainable landing area. The uncertainties for the initial state and the specific fuel consumption are included as error sources during the simulations. Table 3 shows the range of the error for each state and specific fuel consumption. The uncertainties for the initial state are obtained from [19] by imposing the values with the same proportion. The uncertainty for the specific fuel consumption of the main engine $\sigma_{\mathrm{SFC}}$ is around $\% 2$ [20], but in this paper taken as $\% 3$ with a conservative assumption . The cost of attenuating the disturbance and uncertainty is the amount of extra propellant consumed by the main engine. After the Monte Carlo simulations, the results that satisfy the safe landing conditions are filtered leading to nonconvex successful landing regions. The success rate for each corresponding point inside the nonconvex set is evaluated by counting the number of simulations that achieves a successful landing with respect 
Fig. 7: State Error Between the Reference Trajectory and the Simulated Results.
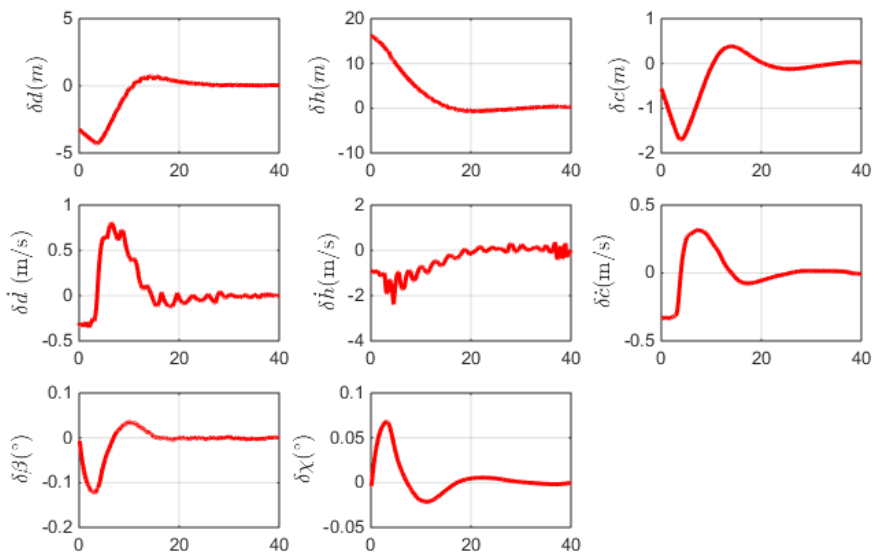

to total number of simulations. The final result is called the successful attainable landing area (SALA) of the lunar lander.

The borderlines that define the nonconvex hull of the successful landing area are obtained using alpha shapes. Finally, an ellipse with the maximum area is fitted inside the nonconvex set to evaluate the ratios of the reachable area, the successful landing area and the area of the ellipse. The parameters of the ellipse are discretized, and the maximum area is computed by a brute force method. Fig. 8 shows the comparison of the reachable landing area without the controller, with the controller and ellipse that fits inside the nonconvex successful landing area.

Fig. 8: Representation of the Reachable Landing Area, Nonconvex 100\% Successful Landing Region and Maximum Area Ellipse

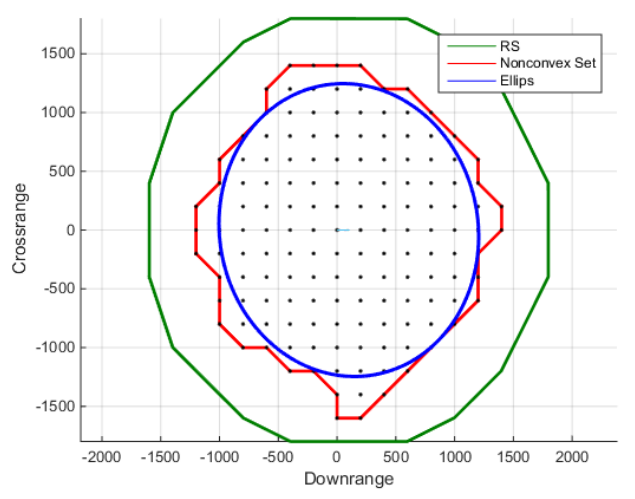


Table 3 summarizes the range of uncertainties and the results obtained from the Monte Carlo simulations. Two scenarios which include respectively $\% 3$ and $\% 5$ uncertainty in the specific fuel consumption together with the initial state errors are denoted as Total(\#1) and Total(\#2). Area ratio denotes the ratio of the area for the reachable set and the nonconvex region. Similarly, the ellipse ratio shows the proportion of the areas for the reachable set and the ellipse.

Table 3 Uncertainties, Results for the Monte Carlo Simulations and Properties of the Maximum Area Ellipses inside the $100 \%$ Successful Landing Region

\begin{tabular}{lllllll}
\hline & Range & Area Ratio & $\begin{array}{l}\text { Ellipse } \\
\text { Ratio }\end{array}$ & Center $(\mathrm{m})$ & Orientation $\left(^{\circ}\right)$ & $\begin{array}{l}\text { Semi-major } \\
\text { Semi-minor } \\
\text { Axis }(\mathrm{m})\end{array}$ \\
\hline$\Delta \dot{d}(\mathrm{~m} / \mathrm{s})$ & {$[-22]$} & 52.75 & 43.65 & {$[1000]$} & 10 & {$[12501100]$} \\
$\Delta \dot{h}(\mathrm{~m} / \mathrm{s})$ & {$[-22]$} & 50.95 & 41.59 & {$[500]$} & 0 & {$[12001100]$} \\
$\Delta \dot{c}(\mathrm{~m} / \mathrm{s})$ & {$[-22]$} & 53.22 & 43.86 & {$[500]$} & 0 & {$[12001150]$} \\
$\Delta d(\mathrm{~m})$ & {$[-3030]$} & 52.02 & 43.89 & {$[1000]$} & 10 & {$[12501100]$} \\
$\Delta h(\mathrm{~m})$ & {$[-3030]$} & 55.49 & 45.56 & {$[1000]$} & 0 & {$[12501150]$} \\
$\Delta c(\mathrm{~m})$ & {$[-3030]$} & 53.09 & 43.76 & {$[500]$} & 0 & {$[12001150]$} \\
$\Delta \beta\left({ }^{\circ}\right)$ & {$[-22]$} & 90.96 & 64.29 & {$[-1000]$} & 20 & {$[15001350]$} \\
$\Delta \chi\left({ }^{\circ}\right)$ & {$[-22]$} & 89.30 & 61.34 & {$[0100]$} & 40 & {$[16001200]$} \\
$\Delta \boldsymbol{m}$ & $\mathbf{\% 5}$ & $\mathbf{4 6 . 0 9}$ & $\mathbf{4 1 . 9 2}$ & {$[\mathbf{1 0 0} \mathbf{0}]$} & $\mathbf{0}$ & {$[\mathbf{1 0 5 0} 1050]$} \\
$\Delta \sigma_{\mathrm{SFC}}$ & $\% 3$ & 46.92 & 41.93 & {$[1000]$} & 0 & {$[12001100]$} \\
$\operatorname{Total}(\# 1)$ & & 29.18 & 34.81 & {$[00]$} & 0 & {$[1000950]$} \\
$\operatorname{Total}(\# 2)$ & & $\mathbf{2 6 . 2 5}$ & $\mathbf{3 1 . 6 6}$ & {$[\mathbf{1 0 0}-\mathbf{5 0}]$} & $\mathbf{0}$ & {$[\mathbf{9 5 0} \mathbf{9 0 0}]$} \\
\hline
\end{tabular}

Table 3 also shows the center, semi-major and semi-minor axis as well as the orientation of the ellipse that fits inside the nonconvex attainable area.

The change of the successful landing area is most sensitive to the mass of the spacecraft or the amount of propellant considering the area ratios. As the uncertainty increases for the initial mass, the points along the boundary of the reachable set is not attainable by the lander during Monte Carlo simulations. Although the tracking controller tracks the reference trajectory, the successful landing condition is not satisfied in some cases. Specific fuel consumption is yet another important parameter affecting the area of the success region. For the cases, where the main engine consumes more propellant per unit thrust, the available propellant is not enough to steer the vehicle to the desired landing point.

On the other hand, the ellipse is not orientated w.r.t. the coordinate axis and the center of the ellipse lies close to the origin in downrange and crossrange coordinates, where the landing maneuver starts.

For the cases Total(\#1) and Total(\#2), all uncertainties are included in the simulations. For each single landing point inside the reachable set, 500 Monte Carlo simulations are performed. The results show that the successful landing area is $26.25 \%$ of the reachable landing area, which is the maximum landing region that can be 
achieved by the lander. The extra propellant consumed for correcting maneuvers result in a substantial decrease of the successful landing area.

\section{Probabilistic Attainable Maps}

Probabilistic attainable areas are obtained for each uncertainty source as illustrated with Fig. 9a-13b. The successful landing area for all uncertainties together are represented with Fig. 14a and Fig. 14b. For each specific case, 500 Monte Carlo simulations are performed using a fixed structure of the feedback controller with the same gains.

As the initial error for the velocity along downrange is introduced, the successful landing region becomes smaller along crossrange, leading to a slender successful landing region as illustrated in Fig. 9a. Similarly, the initial error for the velocity along crossrange results in a smaller successful landing area, with unattainable regions close to the maximum and minimum crossrange of the reachable set in Fig. 10a. The initial error for the rate of altitude has a symmetric behavior in crossrange and downrange coordinates as shown in Fig. 9b. Although initial position error decreases the success rate of the landing, similar attainable landing maps are obtained in Fig. 10b-11b. The results for the position error is also verified from the properties of the ellipses inside the nonconvex $100 \%$ successful landing region in Table 3.

On the other hand, the initial error for the attitude of the spacecraft in $\beta$ and $\chi$ is not very critical in Fig. 12a and Fig. 12b. The success rate of landing decreases slightly for some points on the boundary of the reachable set. However, similar to the discussion for the properties of the ellipses, the mass and the specific fuel consumption of the vehicle is decreasing the success rate of landing considerably in Fig. 13a and Fig. 13b. Finally, for the cases in Total (\#1) and Total (\#2), the success rate of the landing decreases substantially with a symmetrical behaviour as illustrated in Fig. 14a and Fig. 14b.

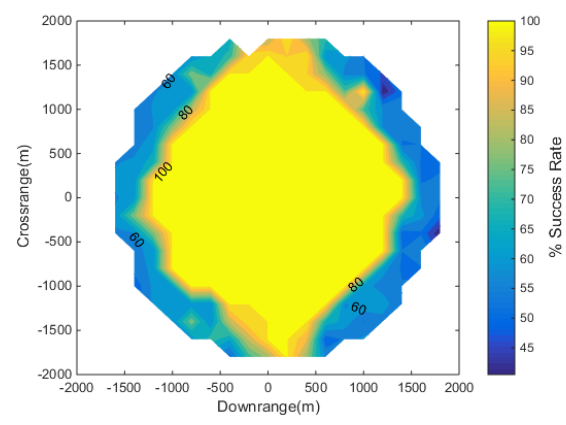

(a)

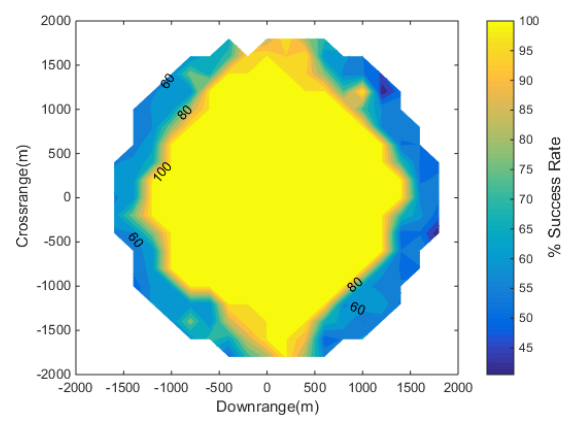

(b)

Fig. 9: SALA with initial error in downrange velocity $\dot{d}$ and altitude rate $\dot{h}$ 


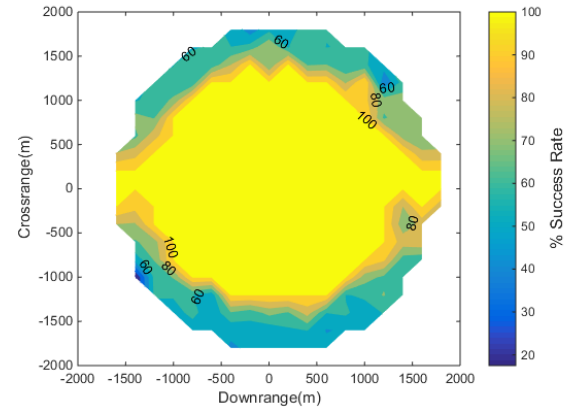

(a)

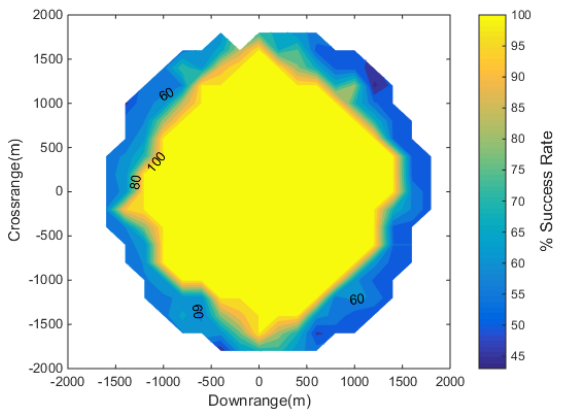

(b)

Fig. 10: SALA with initial error in crossrange velocity $\dot{c}$ and downrange $d$

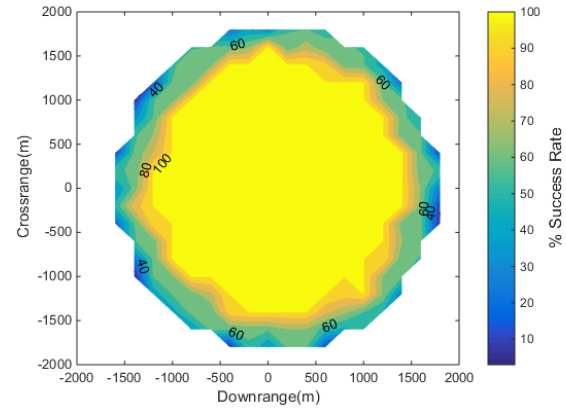

(a)

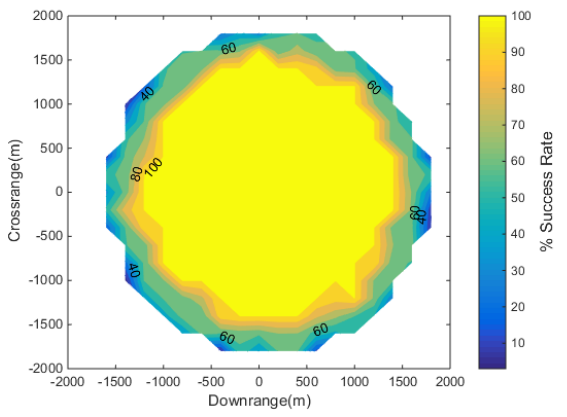

(b)

Fig. 11: SALA with initial error in altitude $h$ and crossrange $c$

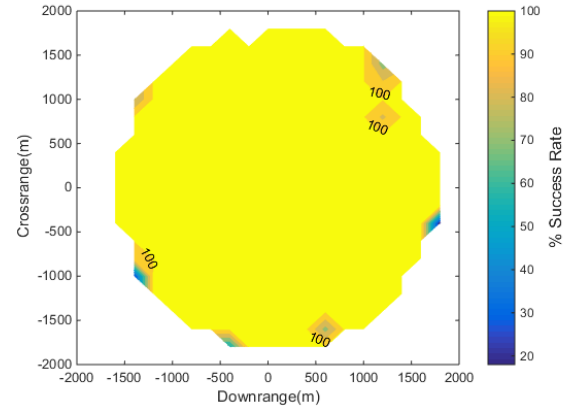

(a)

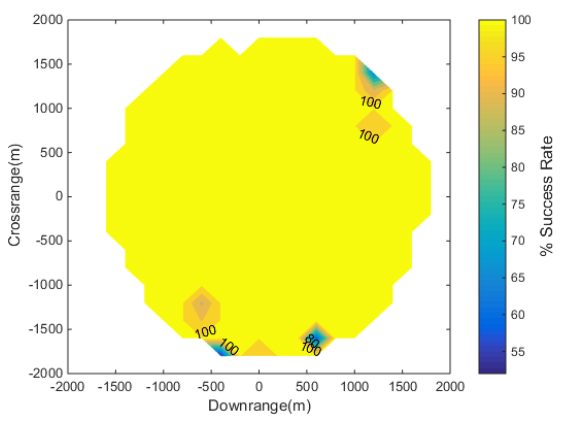

(b)

Fig. 12: SALA with initial error in pitch $\beta$ and yaw $\chi$ 


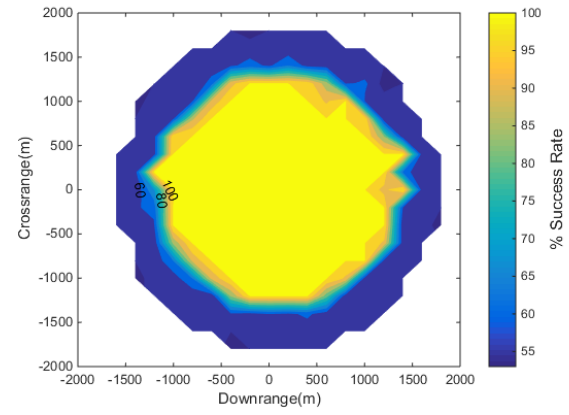

(a)

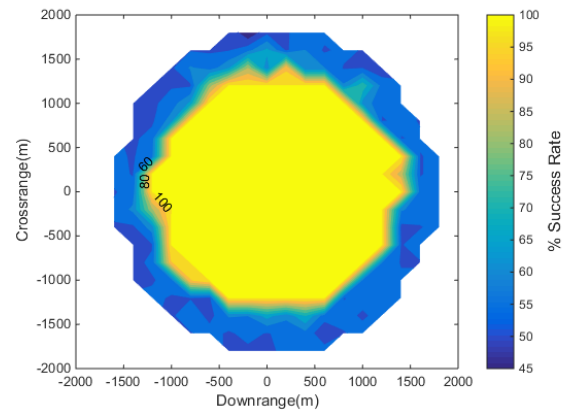

(b)

Fig. 13: SALA with initial error in mass $m$ and specific fuel consumption $\sigma_{S F C}$

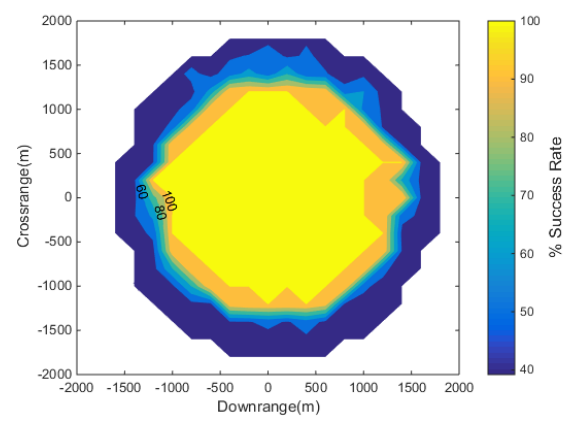

(a)

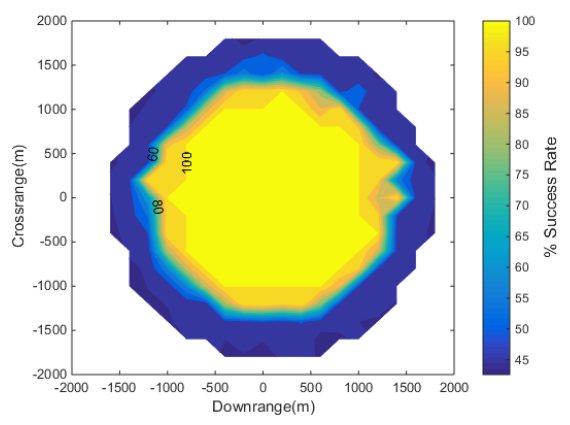

(b)

Fig. 14: SALA for scenario Total(\#1) and Total(\#2)

\section{Conclusions}

The attainable landing area is obtained with reachability analysis, leading to the attainable landing area of the lunar lander for the given mission scenario. A two degree of freedom controller is designed to stabilize the system and attenuate disturbances or initial uncertainties in the states. Monte Carlo simulations are performed to obtain the successful attainable landing area of the lander with probabilistic maps. In order to assess the results of the Monte Carlo simulations, a maximum area ellipse is fitted inside the nonconvex attainable landing area.

The probabilistic maps show the behaviour of the change of success rate with respect to the uncertainties. It is seen that, when uncertainties are introduced, the 
area of the attainable landing region decreases due to the amount of extra propellant consumed to track the reference trajectories in all scenarios. The error for the initial velocity in crossrange and downrange decreases the success rate of the landing starting from extreme points in the downrange and crossrange coordinates respectively. Initial position errors in three orthogonal coordinates have similar probabilistic maps, with similar characteristics of the maximum area ellipse. On the other hand, the initial attitude error has no significant effect on the probabilistic maps.

The lander is most sensitive to changes of the initial mass for a successful landing. The ratio of the attainable area with respect to the successfully attainable area is $46.09 \%$ for the uncertainty in the initial mass. The scenario in Total(\#2) has the smallest attainable landing area, as expected, in which only $26.25 \%$ of the initial reference map is attainable when all uncertainties are introduced in the MC simulations.

The guidance and control algorithms also have an impact on the computed RS for the given dynamical system and uncertainties. The developed method provides a tool to evaluate the performance of the $\mathrm{G} \& \mathrm{C}$ algorithms and optimize the parameters to obtain the maximum landing area w.r.t the reference RS. Future work includes the assessment of different $\mathrm{G} \& \mathrm{C}$ algorithms and the optimization of the parameters for increasing the likelihood of a successful landing.

Acknowledgements This research is supported and funded by DLR (German Aerospace Center), DAAD (German Academic Exchange Service) Research Fellowship Programme and the Excellence Initiative of the German Research Foundation (DFG).

\section{References}

1. R. Baier, C. Büskens, I. A. Chahma, and M. Gerdts. Aproximation of reachable sets by direct solution methods of optimal control problems. Optimization Methods and Software, 22 (3):433-452, 2007.

2. I. Kitsios and J. Lygeros. Aerodynamic envelope computation for safe landing of the hl-20 personnel launch vehicle using hybrid control. In Intelligent Control, 2005. Proceedings of the 2005 IEEE International Symposium on, Mediterrean Conference on Control and Automation, 2005.

3. J. Benito and K. D. Mease. Characterizing the controllable entry states and reachable sites for planetary landing. In 6th International Planetary Probe Workshop, Atlanta, GA, 2008.

4. J. Benito and K. D. Mease. Reachable and controllable sets for planetary entry and landing. Journal of Guidance, Control and Dynamics, 33:2010, 2010.

5. J. P. Aubin and H. Frankowska. Set Valued Analysis. Birkhauser, 1990.

6. I. M. Mitchell. Application of Level Set Methods to Control and Reachability Problems in Continuous and Hybrid Problems. PhD thesis, Standford University, 2002.

7. R. Baier and M. Gerdts. A computational method for non-convex reachable sets using optimal control. In Proceedings of the European Control Conference (ECC) 2009, August 23-26, Budapest, Hungary, 2009. 
8. D. Garg. Advances in Global Pseudospectral Methods for Optimal Control. PhD thesis, University of Florida, 2011.

9. T. Oehlschlägel, S. Theil, H. Krüger, M. Knauer, J. Tietjen, and C. Büskens. Optimal guidance and control of lunar landers with non-throttable main engine. In Advances in Aerospace Guidance, Navigation and Control. Springer Berlin Heidelberg, 2011.

10. D. Garg, M. A. Patterson, C. Francolin, C. L. Darby, G.T. Huntington, W.W. Hager, and A. V. Rao. Direct trajectory optimization and costate estimation offinite-horizon and infinitehorizon optimal control problems using a radau pseudospectral method. Computational Optimization and Applications, 49:335-358, 2011.

11. M. Fahroo. F.and Ross. On discrete-time optimal conditions for pseudospectral methods. In AIAA/AAS Astrodynamics Specialist Conference, Keystone, Colorado, August 2006.

12. G. D. Huntington. Advancement and Analysis of a Gauss Pseudospectral Transcription for Optimal Control Problems. PhD thesis, Massachusetts Institute of Technology, 2007.

13. C. Runge. Über empirische funktionen und die interpolation zwischen äquidistanten ordinaten. Zeitschrift für Mathematik und Physik, 46:224-243, 1901.

14. M. Sagliano, M. Samaan, S. Theil, and E. Mooij. Shefex-3 optimal feedback entry guidance. In AIAA SPACE 2014 Conference and Exposition, August 2014.

15. M. Sagliano. Performance analysis of linear and nonlinear techniques for automatic scaling of discretized control problems. Operations Research Letters, 42:213-216, 2014.

16. M. Sagliano and S. Theil. Hybrid jacobian computation for fast optimal trajectories generation. In AIAA Guidance, Navigation and Control(GNC) Conference, August 2013.

17. Y. E. Arslantas, T. Oehlschlägel, M. Sagliano, S. Theil, and C. Braxmaier. Approximation of attainable landing area of a moon lander by reachability analysis. In 17th International Conference on Hybrid Systems: Computation and Control, 2014.

18. Y. E. Arslantas, T. Oehlschlägel, M. Sagliano, S. Theil, and C. Braxmaier. Safe landing area determination for a moon lander by reachability analysis. In International Astronautical Congress, Toronto, 2014.

19. Murray L. Kerr, Miguel Hagenfeldt, Jose A. Ospina, Jose Miguel Ramn, and Luis F. Penin. Esa lunar lander: Approach phase concept and g\&c performance. In AIAA Guidance, Navigation, and Control (GNC) Conference, Boston, MA, August 19-22 2013.

20. Kenneth J. Davidian, Ronald H. Dieck, and Isaac Chuang. A detailed description of the uncertainty analysis for high area ratio rocket nozzle tests at the nasa lewis research center. Technical report, NASA, 1987. 